Biophysics

\section{Use of deuterated proteins in nuclear magnetic resonance}

\author{
from Paul Rösch
}

Proton nuclear magnetic resonance (NMR) spectroscopy provides information on the tertiary structures of small compounds of biochemical interest such as peptides, small proteins or pieces of DNA. It is also well suited to monitor qualitatively structural changes induced by complex formation of different molecules such as enzymes and their substrate ligands. Attempts to measure the change of ligand conformation on binding to a protein is severely impaired because in most cases proton resonances from the protein tend to obscure all or many of the ligand resonances, especially if the ligand molecule is itself a peptide or a protein. In addition, the magnetic dipolar interactions between ligand and protein protons prevent either conformation from being determined independently, rendering structure determinations of such systems virtually impossible. A recent study by Seeholzer et al. ${ }^{1}$ attempts to distinguish structurally between the inactive and active forms of an enzyme. The authors studied the complex of the eukaryotic calcium-dependent regulatory protein calmodulin and the bee-venom peptide melittin. The calmodulin/melittin complex is probably not physiologically significant, but has potential as a model of the calmodulin/target-protein interaction.

To observe the proton NMR spectrum of the bound peptide Seeholzer et al. ${ }^{1}$ used perdeuterated calmodulin and protonated melittin as complex constituents. This allows the spectrum of the ligand to be observed without interference from the protein resonances. The use of partially or completely deuterated compounds in ${ }^{1} \mathrm{H}$ NMR to observe protonated ligands is by no means a new approach, first publications on the subject appearing as early as 1968 (refs 2,3).

What is novel about the work of Seeholzer et al. is that they used a completely deuterated protein of animal origin. They obtained it in the straightforward way of cloning the bovine calmodulin gene into Escherichia coli and subsequently growing the bacteria in a perdeuterated medium. Because only the signals from the protonated 26-amino-acid peptide melittin appear in the spectrum of its complex with the 148-amino-acid protein calmodulin, Seeholzer et al. ${ }^{\prime}$ could also deduce the spectrum of the protein in the complex by use of difference spectroscopy between the deutero-calmodulin/melittin complex and the fully protonated calmodulin/ melittin complex.
Studies of structural changes induced by complex formation between proteins and their target or substrate molecules are among the most interesting in protein biophysics. In most cases, information on protein-bound molecules is obtained in two ways: either by indirect observation of the influence of complex formation via detection of excess ligand molecule, making use of the fact that the spectrum of the uncomplexed ligand in the presence of the complex-forming molecule is disturbed by the influence of intermediate complex formation; or by monitoring nuclei present

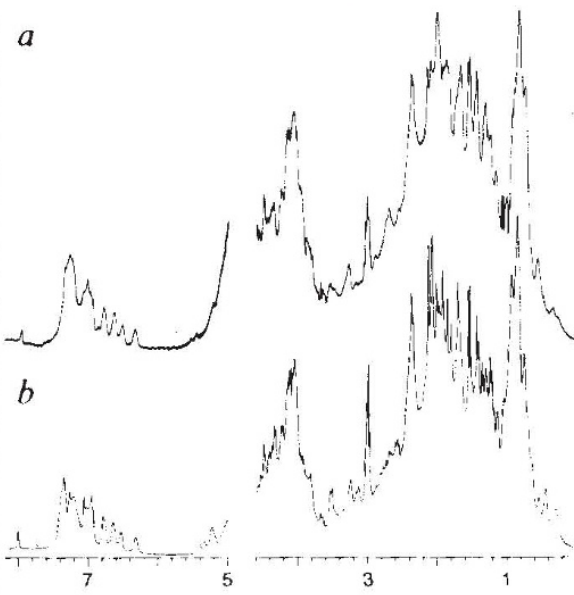

Comparison of ${ }^{1} \mathrm{H}$ NMR spectra of free calmodulin $(a)$ and the calmodulin/ melittin complex $(b)$. (From ref. 1.)

exclusively or predominantly in the ligand, such as ${ }^{31} \mathrm{P}$ in the substrates of phosphoryl-transferring enzymes ${ }^{4}$ or ${ }^{13} \mathrm{C}$, ${ }^{15} \mathrm{~N}$ and ${ }^{19} \mathrm{~F}$ of isotopically labelled or enriched ligand molecules. In fact, one of the co-authors of the publication by Seeholzer et al. ${ }^{1}$, Mildred Cohn, devoted much of her career to observations of ${ }^{31} \mathrm{P}$ NMR spectra of such enzyme-bound substrates, which provided valuable information on protein-ligand interactions.

To gain insight into the structure of protein-bound molecules it is in general necessary to analyse their proton NMR spectra. The observation of such spectra of ligands in the complex can be accomplished as described above. In a way, this procedure is rather similar to the substitution of perdeuterated solvents in NMR (and other branches of spectroscopy) to enhance the observability of sample resonances close to the solvent peaks. As a bonus, one also gets rid of the magnetic dipolar interactions between protein protons and ligand protons, so that only intrapeptide interactions need consideration in the liquid-NMR context. This is very useful for the final structure determination by two-dimensional $\mathrm{NMR}^{5}$ because the ligand can be studied independently.

From the overall appearance of the spectrum of bound melittin ${ }^{1}$ the presence of structured strands in the peptide can be inferred. Melittin is in an extended form as a monomer in $\mathrm{D}_{2} \mathrm{O}$, but assumes more structure in deutero-methanol ${ }^{1,6}$ and when bound to deuterated lipids ${ }^{7}$. As the authors themselves state, a detailed analysis of the structure of calmodulin-bound melittin will only be possible using twodimensional NMR.

Judging from the quality of the spectra, obtaining two-dimensional spectra in $\mathrm{D}_{2} \mathrm{O}$ should be straightforward. It may be even easier than collecting high-quality twodimensional nuclear Overhauser-effect spectra of a peptide of this size free in solution (see the recent discussion in News and Views $\left.{ }^{8}\right)$, because the motional correlation times are longer in the complex. In contrast, obtaining two-dimensional spectra of the ligand involving exchangeable protons in $\mathrm{H}_{2} \mathrm{O}$, which is necessary for the sequential assignment of resonances, poses a problem, because the protein and ligand resonances are expected to overlap in this region. Many resonance assignments have already been made for the free peptide 6 .

Seeholzer et al. present a simple and straightforward application of genetic engineering methods to enhance the effectiveness of NMR. Cloning of calmodulin into $E$. coli was the necessary prerequisite for obtaining the perdeuterated species. Unfortunately, it is not possible to grow every particular strain of bacteria in perdeuterated medium. Neither is it very easy to incorporate specifically all types of amino acids in protonated or deuterated form into bacteria. On the other hand, genetic engineering methods now allow sequence-specific substitution of amino acids at the gene level (site-directed mutagenesis). Spectral assignments of single amino-acid residues and studies of the structural influence of single amino-acid substitutions by NMR should thus be possible. This technique of 'site-directed NMR' holds great promise.

1. Seeholzer, S.H., Cohn, M., Putkey, J.A., Means, A.R. \& Crespi, H.L. Proc. natn. Acad. Sci. U.S.A. 83, 3634 (1986) 2. Markley, J.L., Putter, I. \& Jardetzky, O. Science 161, 1249 (1968).

3. Rosenberg, R.M., Crespi, H.L. \& Katz, J.J. Biochim. bio phys. Acta 175, 31 (1969).

4. Rösch, P. Prog. Nucl. Magn. Reson. Spectrosc. 18, 123 (1986).

5. Bax, A. \& Lerner, L. Science 232, 960 (1986)

6. Lauterwein, J., Brown, L.R. \& Wüthrich, K. Biochem biophys. Acta 622, 219 (1980).

Lauterwein, J., Bösch, C., Brown, L.R. \& Wüthrich, K. Biochim. biophys. Acta 556, 244 (1979)

8. Kabsch, W. \& Rösch, P. Nature News and Views 321, 469 (1986).

Paul Rösch is in the Department of Biophysics, Max-Planck Institute for Medical Research, 6900 Heidelberg 1, FRG. 